\title{
Die Ungeschichtlichkeit des Verräters Judas.
}

\author{
Von Dr. O. Schläger in Cassel.
}

In den "Schriften des neuen Testaments für die Gegenwart erklärt" ${ }^{1}$ bemerkt J. Weiß: „Das sittlich-psychologische Judas-IschariothProblem ist trotz vieler Bemühungen bisher ungelöst. Immer bleiben die Fragen: Wie konnte ein Mann, der längere Zeit unter dem Einfluß Jesu stand, so handeln? und die andere: Wie konnte Jesus diesen Mann unter seine vertrauten Jünger aufnehmen? Eine wissenschaftliche Antwort läßt sich nicht geben; hier muß die Phantasie alles leisten; darum ist dieser Gegenstand die Domäne der Dichter." Wie sehr das Problem Dichter angezogen hat und wie mannigfaltig die Antwort auf die Frage nach dem Warum des Verrats lauten, darüber gibt der Aufsatz von Dr. A. Luther, Jesus und Judas in der Dichtung (Hanau 1910) eine gute Übersicht. Aber auch in die wissenschaftliche Erklärung spielt die phantasievolle Deutung doch bis in die neueste Zeit stark hinein. In Schenkels Bibellexikon 3. Bd. S. 431 konnte Mangold sich noch in folgender Weise "mit annähernder Sicherheit" über Judas aussprechen: ${ }_{n}$ Judas muß zu der Schar derjenigen gehört haben, welche auf den Trost Israels warteten und sich deshalb an. Jesus angeschlossen haben, und Jesus mag ihn in den Kreis der Apostel aufgenommen haben, weil er an ihm neben der Begeisterung für die messianische Wiederherstellung des jüdischen Volkes auch wohl noch andere lobenswerte Eigenschaften bemerkt hatte, eine nicht geringe Energie und ein gewisses administratives Talent, welche der ersten messiasgläubigen Gemeinde zu nicht geringem Nutzen hätte gereichen können. Indes auch die Schatten in Judas' Charakter sind Jesu gewiß nicht verborgen geblieben, eine fleischliche Auffassung des Gottesreiches und eine lohnsüchtige Beteiligung an dessen Jüngerschaft, welche der Schoßsünde des Judas, seiner Habsucht, eine nicht geringe Befriedigung in Aussicht zu stellen schien. Aber gewiß hoffte die Sünderliebe Jesu auch einen Judas noch zum

1 2. Aun., Göttingen 1907, S. 101. 
tüchtigen Werkzeug für den Dienst in seinem Reich heranzubilden ... Die Parabel vom Unkraut unter dem Weizen, die Gleichnisrede von dem Netz mit guten und schlechten Fischen, das ernste Wort über das Ärgernis und ganz spät noch die Rede von dem Mann, der ohne hochzeitliches Kleid beim Festmahl des Königs erscheint - diese Aussprüche Jesu haben alle auch eine allgemeine Bedeutung, sie sind aber doch ebensosehr als ernste Mahnungen für den einen gemeint, der unter seinen Jüngern in Gefahr stand, abtrünnig zu werden. Und ganz ohne Eindruck blieben diese Worte Jesu gewiB nicht, denn lange dauerte der Kampf zwischen Licht und Finsternis bei Judas.“ Das ist alles ganz nett ausgedacht, aber eben auch nur ausgedacht, etwa in der Art, wie ein Prediger eine kurz skizzierte Geschichte mehr oder minder lebensvoll auszumalen sucht. In den Evangelien suchen wir doch vergeblich nach Anhaltspunkten für die meisten dieser Deutungen oder Deuteleien, das meiste ist doch reine Phantasie oder Vermutung, und der Erklärer operiert mit „er muß“, „er mag“ und besonders häufig mit "gewiß“. Auch ein so kritischer Exeget wie Holtzmann scheute sich nicht, in seinem Handkommentar ${ }^{2}$ 1. Bd. S. 277 zur Vorhersagung des Verrats beim Abendmahl zu bemerken: „Jesus hatte ihn mit wachsender Bestimmtheit an seinem veränderten, scheu und trotzig werdenden Verhalten, an seinen geheimen Wegen, an der aufgeregten und gespannten Stimmung erkannt.“ Zu Mt 26, 25 schreibt er: „Judas fragt direkt und frech, ob er gemeint sei." Auch das alles empfinden wir als Versuch, den Verräter und das Verhalten Jesu zu ihm psychologisch begreiflich zu machen. Aber nicht wissenschaftliche Erkenntnis, die sich an die knappen Aussagen der Evangelien hält, sondern ausfüllende dichterische Phantasie führt hier das. Wort. Unser für lebensvolle Wirklichkeit geschärfter Sinn stellt Fragen, die aus den evangelischen Berichten schlechtweg nicht $\mathrm{zu}$ beantworten sind. W. Wrede, der von deutschen Forschern wohl zuletzt sich eingehend mit „Judas Ischarioth in der christlichen Überlieferung " ${ }^{1}$ beschäftigt hat, bemerkt $\mathrm{zu}$ der Vorhersage des Verrats: „Wir hören gar nichts davon, wie die Antwort Jesu auf die Jünger wirkt und was mit Judas geschieht, anscheinend bleibt er beim Mahl ruhig zugegen - das sind Fragen, für die der Evangelist kein Auge hat.“ Und weiter: „Die Frage: Herr, bin ich's? hat für uns etwas so Vertrautes, vielleicht auch so Ergreifendes, daß wir kaum fühlen, wie fremd sie der nüchternen Wirklichkeit ist. Jünger, die sich voller Anhänglichkeit an Jesus bewußt waren,

1 Vorträge und Studien, Tübingen 1907, S. 127-146. 
sollten wirklich meinen, sie seien möglicherweise doch nach einigen Stunden die Verräter?" Steudel ${ }^{1}$ weist auf die Frage hin, ob sich Jesus, wenn er die verräterischen Absichten eines Jüngers wie Judas im voraus erkannte, nicht verpflichtet fühlen mußte, vorher in seelsorgerischer Absicht auf ihn einzuwirken. Er hebt als Unmöglichkeiten hervor: "So läßt der Evangelist ja dann auch den Judas durch sein mit Jesu in die Schüssel Tauchen sich selbst verraten, ohne daß auch nur ein einziger im Jüngerkreis seinem Entsetzen darüber Ausdruck verleiht, daß in ihrer eigenen Mitte der Verräter des geliebten Meisters sich befindet! Und noch unbegreiflicher ist, daß die Elf den Judas unmittelbar nach der Voraussage Jesu, die ihnen doch allen Grund gegeben hätte, auf den als Verräter bezeichneten Jünger ein Auge zu haben, ruhig seines Weges ziehen lassen, wie er zu dem Hohenpriester geht, während. sie selbst Jesum in den Garten Gethsemane begleiten. Unbegreiflich bleibt ja auch, trotz der mancherlei geistreichen und bis auf Goethe zurückführenden Erklärungsversuche, die dafür ausgedacht wurden, die Tat des Judas selbst." Wenn Weizsäcker ${ }^{2}$ in ruhiger Art sagt, es sei immer unbegreiflich gewesen, daß man eines Verräters bedurfte, um sich der Person Jesu zu bemächtigen, und deshalb den Schluß zieht, Jesus müsse (!) sich in den Tagen nach dem Einzug völlig in der Verborgenheit gehalten haben, so gebraucht Kautsky, ${ }^{3}$ um die Unwahrscheinlichkeit, um nicht zu sagen Sinnlosigkeit, eines Verrats klarzumachen, den aus Gegenwartsverhältnissen gewonnenen drastischen Vergleich: „Das wäre ungefähr so, als wenn die Berliner Polizei einen Spitzel besoldete, damit er ihr die.Person bezeichne, die Bebel heiße." Faßt man alle vorliegenden Unwahrscheinlichkeiten und Schwierigkeiten ins Auge, dann versteht man, wie Joël ${ }^{4}$ von dem Rätsel reden konnte, das uns in der unmöglichen Figur des Judas aufgegeben ist. Wrede nennt den Kern des Tatsächlichen in den Nachrichten des NTs über den Jünger äußerst gering, der weitaus größte Teil sei schon Legende. Er findet es auch „eigentlich nicht befremdlich, daß man auch die Tatsache des Verrats selbst in Zweifel gezogen hat". Aber doch lehnt er die von G. Volkmar vorgebrachte Meinung $a b$, Judas sei eigentlich das Volk Juda, und der Gedanke, das Volk Juda habe Jesus seinen Feinden überliefert, habe sich umgesetzt in die

1. Im Kampf um die Christusmythe, Jena 1910, S. 46.

2 Untersuchungen über die evangel. Geschichte, S. 533.

3 Der Ursprung des Christentums, Stuttgart 1908, S. 388.

4 Blicke in die Religionsgeschichte zu Anfang des 2. Jahrhdts., 2. Abteilung, Breslau 1883, S. 149. 
Vorstellung, ein Jünger namens Judas habe ihn verraten. Wrede nennt das eine schwerlich annehmbare Erklärung und ruft aus: Nein, der Verrat des Judas wird eine geschichtliche Tatsache sein, weil er als Sage ganz unverständlich wäre. Auch ein so radikaler Kritiker wie W. Brandt hält in seiner Evangelischen Geschichte an der. Existenz eines Verräters und der Tatsache des Verrats fest. Er meint: ${ }^{1}$ „Wir dürfen getrost behaupten: schon der erste, der die Geschichte Jesu zu schreiben unternahm, würde selber es nicht geglaubt haben, wenn die Sache nicht von ganz unverdächtiger Seite aufs bestimmteste überliefert gewesen wäre." Demgegenüber fehlt es aber doch auch nicht an Stimmen, die sich wieder für die wohl nie ernst ins Auge gefaßte und fast vergessene Ansicht G. Volkmars aussprechen, daß Judas der Repräsentant des Volkes Juda sei. Volkmar setzt seine Ansicht auseinander in der "Religion Jesu“, Leipzig 1857, S. $260 \mathrm{ff}$. und im "Markus“, Zürich 1876, S. 555. W. B. Smith widmet dem Judas Ischarioth einen Abschnitt in seinem Buche "Ecce Deus“, Jena 1911, S. 295-309, worin er besonders den Namen Ischarioth zu erklären versucht. J. M. Robertson behandelt den "Mythos von Judas Ischarioth" in seinen "Evangelienmythen“, S. $108 \mathrm{ff}$. Auch Feigel trägt starke Bedenken, die Person' des Judas für geschichtlich zu halten, in seinem Buche: Der Einfluß des Weissagungsbeweises und anderer Motive auf die Leidensgeschichte (Tübingen 1910), S. 48ff., 95, 114. In der französischen Wochenschrift "Les droits de l'homme“ vom 23. April 1911 hat der Rabbiner Louis Germain Lévy einen Aufsatz geschrieben: Que Judas le Traître n'a jamais existé. Alle Einzelheiten der Verratslegende sind aus alttestamentlichen Weissagungen erwachsen, wie er annimmt. Der Beachtung wert ist auch eine kleine Broschüre von G. Marquardt, die merkwürdigerweise Wrede nicht zugänglich gewesen ist: Der Verrat des Judas Ischarioth - eine Sage, erschienen als Heft 30 der „Kleinen Studien" im Verlage von August Schupp in München ohne Angabe des Jahres. Vielleicht gelingt es der folgenden Untersuchung, durch Betonung schon geäußerter Bedenken und durch Hinweis auf einige wenig bekannte Tatsachen der Nichtexistenz eines Verräters Judas zu größerer Anerkennung 'zu verhelfen.

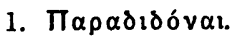

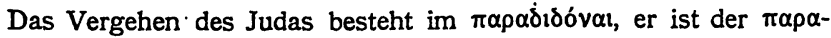

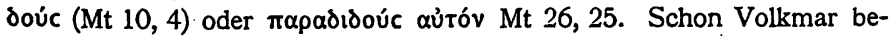

1 Evangel. Geschichte, Leipzig 1893, S. 18. 


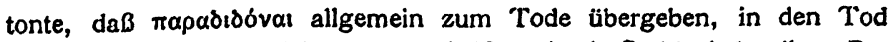
bringen und nicht gleich verraten heiBt. Auch Smith hebt diese Bedeutung stark hervor. Er sagt: „Nun kann zweifelsohne jemand verräterischerweisc ausliefern, eben wie er in verräterischer Absicht küssen oder umarmen oder schreiben oder sprechen oder auch noch vieles andere anstellen kann. Aber keineswegs wird damit von vornherein behauptet, daß küssen, umarmen, schreiben, reden immer sogleich verraten bedeutet. Mithin ist es in keinem der angezogenen Beispiele der deckende Ausdruck, wenn man das Wort mit verraten übersetzte. Insoweit der ,Nebengedanke' des Verrates hereinspielt, liegt das in den Begleitumständen des besonders gearteten Einzelfalles begründet, nicht aber im eigentlichen Sinn des Wortes, der ganz einfach ist: ,überliefern'." In den meisten Fällen übersetzt Luther das Wort mit überantworten, Weizsäcker mit ausliefern. So, wenn es in der Bergpredigt

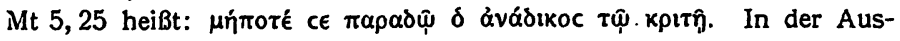
sendungsrede Mt 10,17 sagt Jesus zu den Jüngern: $\pi \alpha \rho \alpha \delta$ ẃcoucıv rà $\rho$

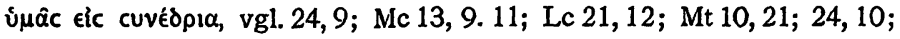
Mc 13,12; Lc 21,16; Mt 18, 34; 24, 9.10. Von Paulus heißt es Act 8, 3,

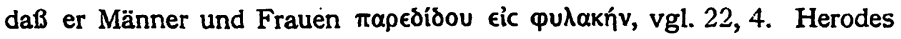
liefert Petrus den Wächtern aus, 12, 4. Agabos weissagt dem Paulus,

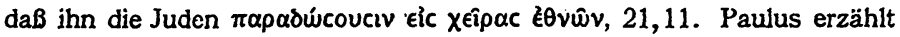

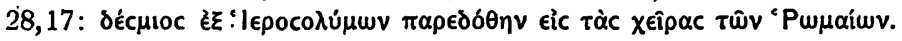
Auch von Johannes dem Täufer heißt es $\cdot$ Mt 4,12, vgl. Mc 1, 14: $\pi \alpha p \in \delta o ́ \theta \eta$. In allen diesen Fällen denkt natürlich kein Leser an verraten, wofür ja die griechische Sprache auch das eigene Wort $\pi$ podrdóval hat. Eînmal, aber auch nur an dieser einen Stelle Lc 6,16, heißt

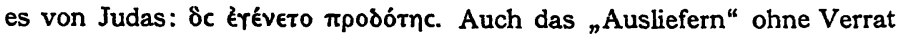
ist eine Schlechtigkeit. So heißt es in der Petrusapokalypse 12, 27 von einigen, die in der Qual sind: oũtol dè ḣcav oi dıúzavtec toùc di-

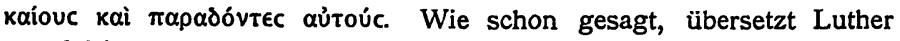

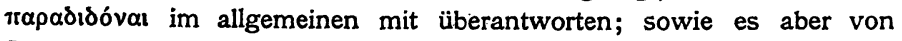
Judas gebraucht wird, nimmt er das Wort verraten. Auch Weizsäcker verfährt im allgemeinen so. Nur an einigen Stellen bleibt er bei ausliefern, wo Luther verraten sagt. . So heißt es bei Luther Mit 26, 15: ich will ihn euch verraten, bei Weizsäcker: was wollt ihr mir geben, daB ich ihn euch ausliefere? Luther sagt Mt 26, 46: siehe, er ist da, der mich verrät, Weizsäcker: siehe, der mich ausliefert, ist da. In der "Vergleichenden .Übersicht der vier Evangelien “ von S. E. Verus (Leipzig 1899, Verlag von P. van Dyk) wird mit peinlicher Genauigkeit jedesmal „überliefern“ gebraucht. Es ist eben nicht gleichgültig, daß 
Tapadidóvai eigentlich nur ausliefern heißt. Die bestimmte Nuance nach der schlechteren Seite hin hat sich vielleicht erst nach und nach herausgebildet, wie auch Volkmar meinte: Der Verräter mag erst aus einer solchen Deutung später hervorgebildet sein.

\section{Das Judenvolk, der Jude als Verräter.}

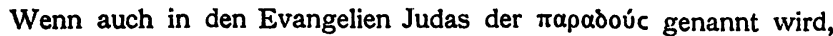
so fehlt es andrerseits doch auch nicht an Aussagen, die dem ganzen Volk der Juden oder wenigstens den führenden Kreisen die Schuld des

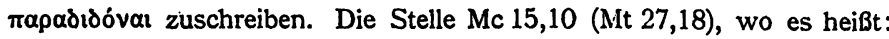

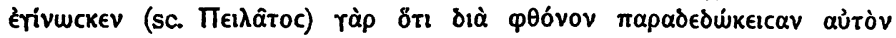

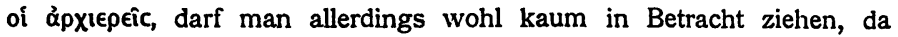
auch bei einem Verrat des Judas die Hohenpriester doch die treibende Kraft, die intellektuellen Urheber sind. Beachtenswert ist nur wieder

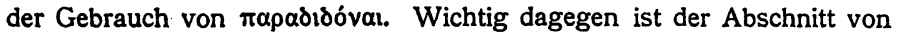
den Emmausjüngern, die dem vermeintlichen Fremdling erzählen, ötwc

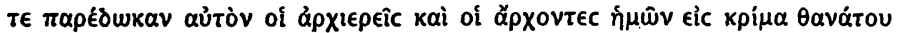
kaì Ectaúpwcav aủróv, Lc 24,20. Ist es nur die Kürze der Darstellung, die diese Mittelursache des Todes Jesu, den Verrat des Judas, übergangen hat, oder hat dieser doch für sich alleinstehende Abschnitt noch nicht die Schuld eines' Individuums Judas gekannt und macht nur das Volk in seinen Führern für den Tod Jesu verantwortlich? Die zweite Annahme, daß nicht ein Ubergehen, sondern ein Nichtwissen um die Tat eines Individuums Judas vorliegt, wird durch die Aussagen der Apostelgeschichte wahrscheinlich. Von Judas ist in diesem Buche nur in dem Abschnitt 1,15-26 die Rede. Über die Berichte über das Ende des Judas urteilt Wrede: „Nach allem ist also der Matthäusbericht völlig sagenhaft. Die Andeutungen der Apostelgeschichte sind aber schwerlich günstiger zu beurteilen." Wir haben jedenfalls einen späten, recht wenig glaubwürdigen Bericht vor uns. Van Manen ${ }^{1}$ erklärt ihn für ein den Zusammenhang unterbrechendes Stück von einer andern Hand als der, die das unmittelbar Vorhergehende und Folgende schrieb. Jedenfalls wird Judas mit keinem Sterbenswörtchen wieder in der Apostelgeschichte erwähnt. Dagegen wird das gesamte Volk der Juden des öfteren hart angeklagt. In der

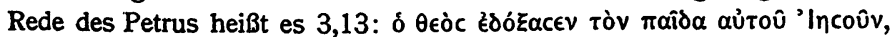

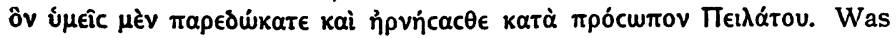

1 Paulus I, De Handelingen der Apostelen, Leiden, 1890, S. 46. 
in den Evangclien zwei einzelnen Personen zugeschoben wird, das

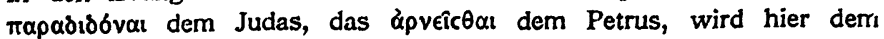
ganzen Judenvolk von Jerusalem zur Last gelegt. Ebenso wird in der Rede des Stephanus 7,52 das Volk gescholten: oũ (sc. toû dıkaíou)

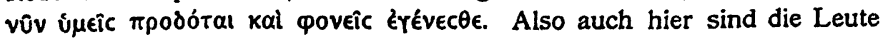

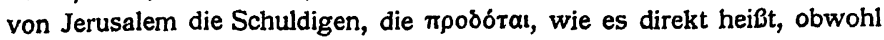
an einen "Verrat" im eigentlichen Sinn gar nicht gedacht werden kann. Auch 2, 23 werden die israelitischen Männer gescholten, daß sie Jesus

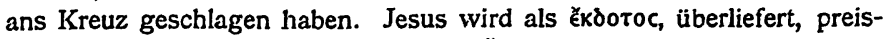
gegeben bezeichnet, aber ein einzelner Überlieferer wird auch hier nicht genannt. Sooft von der Schuld des Volkes die Rede ist, z. B. noch 4,$10 ; 5,30 ; 10,39 ; 13,28$, wird doch nie ein Verrat und ein Verräter erwähnt. Nun kann man sagen, daß die Nichterwähnung des Judas noch kein Beweis für seine Nichtexistenz sei. Gewiß, aber wenn der Verrat des Judas es erst ermöglicht hätte, daß die Oberen sich des Jesus bemächtigten, wenn eine so greuliche Sünde des häßlichsten Verrates geschehen wäre, so lag es nahe, diesen abscheulichen Verbrecher zu erwähnen. Da der Verfasser der Apostelgeschichte heftige Anklagen nur gegen das Volk oder seine Führer erhebt, so scheint es doch so, als ob in seinem Bewußtsein ein einzelner Verräter gar keine Rolle spielt.

\section{Die paulinischen Briefe}

und die alte christliche Literatur.

Auffallend und schwerwiegend ist es, daß die paulinischen Briefe nie von einem Verrat und einem Verräter sprechen. J. Weiß," von dem Bestreben geleitet, den Apostel Paulus möglichst viel von einem Leben Jesu wissen $\mathrm{zu}$ lassen, liest allerdings aus den Worten 1 Kor 11,23 viel heraus. „Sie reden von dem, Verrat" - Paulus weiß also etwas von dem Verräter, von der Verhaftung." Aber dazu ist zu bemerken, daß das $\pi \alpha \rho \in \delta i \delta \in \tau o$ nicht $z u$ übersetzen ist: da er verraten ward, sondern: da er überliefert ward, und daß das Verbum im Passivum steht, also nicht ohne weiteres „an einen Verräter" zu denken ist. Es ist eben nur ganz allgemein gesagt: als er überliefert wurde, und es ist sehr gut möglich zu ergänzen: von den Hohenpriestern und Obersten. Die Behauptung, Paulus weiß etwas von dem Verräter, ist jedenfalls viel zu kühn und unvorsichtig, der Wunsch ist hier der Vater des Gedankens ge-

1 Paulus und Jesus, Berlin 1909, S. 11. 
wesen. ${ }^{1}$ Im übrigen will der Verfasser nicht verschweigen, daß er den Abschnitt 1 Kor 11, 23-32, abgesehen von vielen andern Gründen, gerade wegen seiner in den paulinischen Briefen nur ganz vereinzelt vorkommenden Beziehung auf eine Tatsache aus dem Leben Jesu für eine Interpolation aus späterer Zeit hält. Auch wenn also in 11,23 ein direkter Verrat vorausgesetzt sein sollte, so weiß deshalb Paulus, der Verfasser des ersten Korintherbriefs, doch noch nichts von einem Verräter Judas. Als die Interpolation eingeschoben wurde, mochte die Legende von einem einzelnen Verräter schon bestehen oder in der Entstehung begriffen sein.

Dafür, daß Paulus einen Verräter Judas nicht gekannt hat, hat Volkmar sich auf 1 Kor 15,4 berufen, wonach Christus den „Zwölfen“ erschienen ist. Judas muß also $\mathrm{zu}$ den Zwölfen gehört haben. Aber dagegen kann man sagen, daß der Ausdruck die "Zwölf“ als ein feststehender terminus technicus für den engeren Jüngerkreis gebraucht sei. Ferner aber unterliegt der ganze Abschnitt 15, 3-8 dem Verdacht, überarbeitet $\dot{z} u$ sein. Wenigstens findet sich sonst in den paulinischen

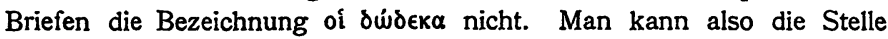
nicht gerade besonders ausnutzen für die Behauptung, daß Paulus nichts von' 'einem Verräter Judas gewußt habe. Sie ergibt sich aus dem Gesamtinhalt der Briefe. Unkenntnis von einem Verrat des Judas findet sich überhaupt in der altchristlichen Literatur. Die apostolischen Väter, die Didache, Justinus, Aristides erwähnen ihn nirgends. Und

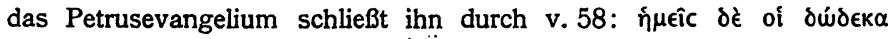

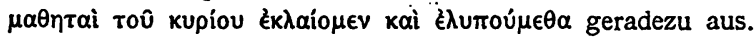

\section{Deutung der Figur des Judas.}

Sollte es bei diesem Tatbestande so unwahrscheinlich sein, die Gestalt des Judas überhaupt für eine Person der Legende zu halten, für eine Verkörperung des jüdischen Volkes, das den Christus der römischen Obrigkeit zum Tode überlieferte? Es ist nicht recht einzusehen, warum diese-Auffassung, um mit Wrede zu reden, eine schwer annehmbare Erklärung sein soll. Die von uns angeführten Stellen der Apostelgeschichte klagen doch das Volk an, und es war dann nur ein Schritt, das Volk der Juden in einem einzelnen Juden, einem Judas, darzustellen.

1 Auch Feigel, Der Einfluß des Weissagungsbeweises und anderer Motive auf die Leidensgeschichte, Tübingen 1910, merkt auf S. 49 zu 1 Kor 11, 23 an: Hier ist nicht zu übersetzen: in der Nacht, da er verraten ward ${ }^{\alpha}$, und daraus etwa zu schließen, daß schon Paulus vom Judasverrat wisse. 
Robertson sucht die Person des Judas als eine Figur aus einem Mysterienschauspiel zu erklären. Er sagt in seiner Short History of Christianity:1 "Judas, der in keinem der Briefe erwähnt wird und dessen sagenhafter Verrat weder in dem kürzlich entdeckten Petrusevangelium noch in der pseudopaulinischen Bezugnahme auf die ,Zwölf ${ }^{6}$ vorkommt, ist eine späte Schöpfung. Wahrscheinlich hat er zuerst als ein einfacher Judaios, als ein Jude, in einem frühchristlichen Mysterienspiel von der Kreuzigung und Auferstehung Gestalt gewonnen ... Die Verratsgeschichte in den Evangelien ist jedenfalls erdichtet, und ihre Existenz läßt sich am besten durch die Beobachtung erklären, daß solch ein Mysterium, das unter den Heiden entstand, ebenso einen Juden als Verräter des Herrn darstellte, wie die Zwölf als Männer galten, die ihren Meister verließen. Der Beutel zur Aufbewahrung des Blutgeldes wurde eine dramatische Zutat und gab zu der Meinung Anlaß, daß Judas der Schatzmeister der Apostelgeschichte war." Auch Lublinski verzichtet darauf, den Verrat des Judas irgendwie historischrationalistisch $z \mathrm{u}$ erklären, da wir alle Motive $\mathrm{zu}$ dem Frevel von uns aus in die Evangelien hineintragen müßten. Er schreibt: ${ }^{2}$ „So bleibt uns nur der Ausweg, daß der Verrat des Judas von einer irrationalen Wesensart ist und allein als ein umgekehrtes Wunder, als Eingriff des Bösen, begreiflich erscheint. Lukas sagt mit dürren Worten, daß der Satan in Judas hineinfuhr, und bei Johannes ist es sogar Christus selbst, der ein Stück Brot in Wein taucht und es dem Judas zu verzehren gibt, worauf in diesen der Böse eindringt. und inn zum Verrat verleitet. Wenn wir uns nun besinnen, daß der Dämon nach der erkenntniskritischen Mythologie des Altertums das eigentliche Wesen eines Menschen ausmacht, so ergibt sich für uns, daß Judas jetzt gar nicht mehr Judas ist, sondern der Satan, der König der Finsternis. Er wird Judas genannt, weil er durch dieses Synonym als Vertreter des gottesmörderischen jüdischen Volkes bezeichnet werden sollte. Diese Hinterabsicht ist nicht $\mathrm{zu}$ bestreiten, aber sie erscheint doch nicht als die einzige Ursache der Vermenschlichung des Satans, des Mörders von Anbeginn. Sobald Christus zum Menschen wurde, mußte der Böse ihm schließlich einmal in gleicher Gestalt entgegentreten, und er hat dadurch entschieden gewonnen. $\mathrm{Er}$ ist nun zum Typus des großen Frevlers und des großen Reuigen geworden, der über sich selber das Todesurteil verhängt. Wahrlich ein großes Wunder dieser Vermenschlichungskunst selbst gegenüber dem Teufel.“

1 London, Watts \& Co. 1902, S. 21.

2 Das werdende Dogma vom Leben Jesu, Jena 1910, S. 145 f. 
Derartige Gedanken sind doch eine durchaus annehmbare Erklärung für die Entstehung der Judasfigur. Mehr als eine solche großzügige Ėrklärung darf man bei einer solchen Frage natürlich nicht erwarten. Sie bildet dann aber doch ein positives Gegenstück zu dem viel leichter zu erbringenden Nachweis der psychologischen Unwahrscheinlichkeiten und Unstimmigkeiten und der mangelhaften geschichtlichen Bezeugung der Verratsgeschichte. So braucht man nicht mit Wrede zu sagen: Der Verrat des Judas wird eine geschichtliche Tatsache sein, weil er als Sage ganz unverständlich wäre, sondern darf getrost behaupten: Der Verrat des Judas ist keine geschichtliche Tatsache, sondern Sage oder Legende. oder, wenn man will, Mythos. 